\title{
Many-body Coulomb gauge exotic and charmed hybrids
}

\author{
Felipe J. Llanes-Estrada, Stephen R. Cotanch \\ Department of Physics, North Carolina State University, Raleigh, NC 27695-8202, USA \\ Received 1 February 2001; accepted 14 February 2001 \\ Editor: H. Georgi
}

\begin{abstract}
Utilizing an effective QCD Coulomb gauge Hamiltonian with linear confinement specified by lattice, we report a relativistic many-body calculation for the light exotic and charmed hybrid mesons. The Hamiltonian successfully describes both quark and gluon sectors, with vacuum and quasiparticle properties generated by a BCS transformation and more elaborate TDA and RPA diagonalizations for the meson $(q \bar{q})$ and glueball $(g g)$ masses. Hybrids entail a computationally intense relativistic three quasiparticle $(q \bar{q} g)$ calculation with the 9-dimensional Hamiltonian matrix elements evaluated variationally by Monte Carlo techniques. Our new TDA (RPA) spectrum for the nonexotic $1^{--}$charmed ( $c \bar{c}$ and $c \bar{c} g$ ) system provides an explanation for the overpopulation of the observed $J / \psi$ states. For the important $1^{-+}$light exotic channel we obtain hybrid masses above $2 \mathrm{GeV}$, in broad agreement with lattice and flux tube models, indicating that the recently observed resonances at 1.4 and $1.6 \mathrm{GeV}$ are of different, perhaps four quark, structure. (๑) 2001 Published by Elsevier Science B.V.
\end{abstract}

PACS: 12.39.Mk; 12.39.Pn; 12.39.Ki; 12.40.Yx

Keywords: Hybrid mesons; Exotic states; Charmonium; Effective Hamiltonian; QCD Coulomb gauge; Potential models

Exotic hybrids, hadrons with quantum numbers not possible in simple $q \bar{q}$ or $q q q$ quark models, have been an elusive, yet signature prediction of Quantum Chromodynamics (QCD). It was therefore quite natural that the recent observation by the E852 collaboration [1] of two exotic $J^{P C}=1^{-+}$states with masses 1.4 and $1.6 \mathrm{GeV}$ would attract widespread interest. Since these states have isospin $I=1$ they can not be glueballs (oddballs) and would initially appear to be viable hybrid meson candidates, especially since vintage bag model calculations [2] predict exotic excitations with explicit gluonic degrees of freedom in this mass range. However, the detailed structure of these states remains uncertain since most contemporary theoretical studies,

E-mail address: cotanch@ncsu.edu (S.R. Cotanch). such as lattice gauge [3-6], flux tube [7,8], QCD sum rule [9], perturbative non-relativistic QCD [10] and constituent models [11] have focused on heavy quark hybrids. The only two modern light quark hybrid calculations, a lattice gauge [4] and flux tube [8], have further compounded this uncertainty by predicting the lightest exotic hybrid mass to be about $2.0 \mathrm{GeV}-$ significantly above the observed $1^{-+}$states. Because the bag model results are rather dated and lattice calculations are less accurate for light quarks due to extrapolation, it is important to have an additional, alternative hybrid prediction. The purpose of this Letter is to determine if these exotic states can indeed be interpreted as hybrids within a relativistic many-body constituent approach that has successfully described both conventional meson [12,13] and glueball [14] systems. 
Our starting point is the QCD Coulomb gauge Hamiltonian (see, for example, Ref. [15]) which we simplify to a form amenable for many body calculations

$$
\begin{aligned}
H= & \int d \mathbf{x} \Psi^{\dagger}(\mathbf{x})(-i \alpha \cdot \nabla+\beta m) \Psi(\mathbf{x}) \\
& +\operatorname{Tr} \int d \mathbf{x}\left(\boldsymbol{\Pi}^{a} \cdot \boldsymbol{\Pi}^{a}+\mathbf{B}_{A}^{a} \cdot \mathbf{B}_{A}^{a}\right) \\
& -\frac{1}{2} \int d \mathbf{x} d \mathbf{y} \rho^{a}(\mathbf{x}) V(|\mathbf{x}-\mathbf{y}|) \rho^{a}(\mathbf{y}) .
\end{aligned}
$$

Here $\Psi$ and $\mathbf{A}$, are the respective quark and gluon fields, $\mathbf{B}_{A}^{a}=\nabla \times \mathbf{A}^{a}$, and $\rho^{a}=\Psi^{\dagger} T^{a} \Psi+f^{a b c} \mathbf{A}^{b} \cdot \boldsymbol{\Pi}^{c}$ is the quark plus gluon color density. The current quark mass, $m$, is assigned the values, $m_{u}=m_{d}=$ $5 \mathrm{MeV}$ and $m_{c}=1200 \mathrm{MeV}$ for the $u, d$ and $c$ flavors, respectively. Confinement and leading canonical interactions are represented by the instantaneous potential, $V=-\frac{\alpha_{s}}{r}+\sigma r$, with $\alpha_{s}=0.2$, and, $\sigma=$ $0.135 \mathrm{GeV}^{2}$, as determined by the string tension from lattice and Regge fits. We also use a cut-off parameter $\Lambda=4-5 \mathrm{GeV}$ to regularize the logarithmic divergent term in the mass gap equation. The model parameters $\sigma, \alpha_{s}$ and $\Lambda$ are commensurate with our previous pure quark [12,13] and gluon [14] applications which produced reasonable hadronic description including the Regge trajectory slopes for the mesons (e.g., $\rho$ tower) and glueballs (pomeron) [16].

Next we proceed to the many-body diagonalizations but first perform a canonical transformation (BCS rotation) to a new quasiparticle basis

$$
\begin{aligned}
& \alpha_{i}^{a}(\mathbf{k})=\cosh \Theta_{k} a_{i}^{a}(\mathbf{k})+\sinh \Theta_{k} a_{i}^{a \dagger}(-\mathbf{k}), \\
& B_{c \lambda}(\mathbf{k})=\cos \frac{\theta_{k}}{2} b_{c \lambda}(\mathbf{k})-\lambda \sin \frac{\theta_{k}}{2} d_{c \lambda}^{\dagger}(-\mathbf{k}), \\
& D_{c \lambda}(-\mathbf{k})=\cos \frac{\theta_{k}}{2} d_{c \lambda}(-\mathbf{k})+\lambda \sin \frac{\theta_{k}}{2} b_{c \lambda}^{\dagger}(\mathbf{k}),
\end{aligned}
$$

where $\Theta_{k}, \theta_{k} / 2$ are the BCS angles, further specified below, and $a(\alpha), b(B)$ and $d(D)$ are bare (dressed) gluon, quark and antiquark Fock operators, respectively. The indices $a=1,2, \ldots, 8$ and $c=1,2,3$ denote color while $\lambda$ represents spin projection. The new field expansions are

$$
\begin{aligned}
A_{i}^{a}(\mathbf{x})=\int & \frac{d \mathbf{k}}{(2 \pi)^{3}} \frac{1}{\sqrt{2 \omega_{k}}} \\
& \times\left[\alpha_{i}^{a}(\mathbf{k})+\alpha_{i}^{a^{\dagger}}(-\mathbf{k})\right] e^{i \mathbf{k} \cdot \mathbf{x}},
\end{aligned}
$$

$$
\begin{aligned}
\Pi_{i}^{a}(\mathbf{x})=-i \int & \frac{d \mathbf{k}}{(2 \pi)^{3}} \sqrt{\frac{\omega_{k}}{2}} \\
& \times\left[\alpha_{i}^{a}(\mathbf{k})-\alpha_{i}^{a^{\dagger}}(-\mathbf{k})\right] e^{i \mathbf{k} \cdot \mathbf{x}},
\end{aligned}
$$

for the gluon fields and

$$
\begin{aligned}
\Psi(\mathbf{x})= & \sum_{c \lambda} \int \frac{d \mathbf{k}}{(2 \pi)^{3}} \\
& \times\left[U_{c \lambda}(\mathbf{k}) B_{c \lambda}(\mathbf{k})+V_{c \lambda}(-\mathbf{k}) D_{c \lambda}^{\dagger}(-\mathbf{k})\right] e^{i \mathbf{k} \cdot \mathbf{x}},
\end{aligned}
$$

for the fermion field. The rotated Dirac spinors are given in terms of the Pauli spinors, $\chi$,

$$
\begin{aligned}
& U_{c \lambda}(\mathbf{k})=\frac{1}{\sqrt{2}}\left[\begin{array}{c}
\sqrt{1+\sin \phi_{k}} \chi_{c \lambda} \\
\sqrt{1-\sin \phi_{k}} \sigma \cdot \hat{\mathbf{k}} \chi_{c \lambda}
\end{array}\right], \\
& V_{c \lambda}(\mathbf{k})=\frac{1}{\sqrt{2}}\left[\begin{array}{c}
-\sqrt{1-\sin \phi_{k}} \boldsymbol{\sigma} \cdot \hat{\mathbf{k}} \chi_{c \lambda} \\
\sqrt{1+\sin \phi_{k}} \chi_{c \lambda}
\end{array}\right] .
\end{aligned}
$$

An improved, nontrivial vacuum, $|\Omega\rangle$, is then obtained by minimizing the ground state expectation value of the Hamiltonian variationally with respect to the BCS angles. Actually, the specific variational parameters are the quark gap angle, $\phi_{k}$, related to the BCS angle by $\tan \left(\phi_{k}-\theta_{k}\right)=m / k$, and the gluon self-energy, $\omega_{k}$, satisfying $\omega_{k}=k e^{-2 \Theta_{k}}$. This generates a quark and gluon gap equation (equivalent to the Schwinger-Dyson equation) yielding mass gaps of about $100 \mathrm{MeV}$ for the $u / d$ quarks and $800 \mathrm{MeV}$ for the gluon. The BCS vacuum contains quark and gluon condensates (Cooper pairs) in reasonable agreement with QCD sum rules. For more complete details consult Refs. [12-14].

Through extensive studies [12-14,16] we ascertained that the two body sector is adequately described by the Tamm-Dancoff approximation (TDA) in which a glueball is represented by Fock states $g^{\dagger} g^{\dagger}|\Omega\rangle$ and mesons by $q^{\dagger} \bar{q}^{\dagger}|\Omega\rangle$. The notable exception is the light pseudoscalar sector, where a more sophisticated, collective approximation (Random Phase approximation or RPA) is needed to correctly reproduce the Goldstone boson nature of the pion due to spontaneous chiral symmetry breaking by our BCS vacuum. This is also further documented in Refs. [12,13].

Finally we perform the appropriate color contraction of octet quark and gluon states to obtain a color 


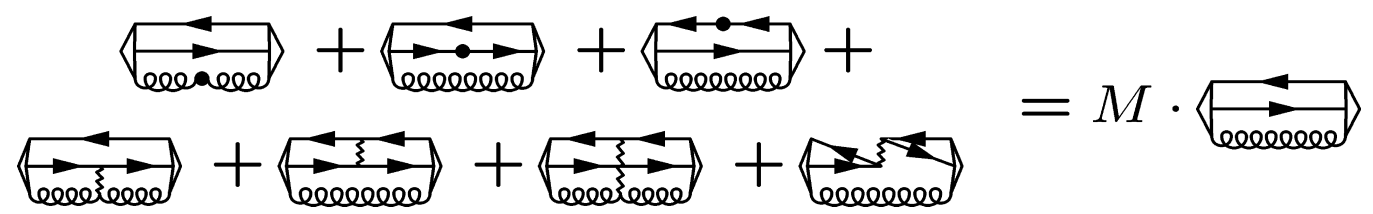

Fig. 1. One (dot) and two-body (waves) TDA matrix element for hybrid mesons with one constituent gluon. Note the $q \bar{q}$ annihilation two-body diagram.

singlet hybrid meson

$$
\begin{aligned}
\mid \text { hybrid }\rangle & =q^{\dagger} \bar{q}^{\dagger} g^{\dagger}|\Omega\rangle \\
& \equiv\left[\left[q^{\dagger} \bigotimes \bar{q}^{\dagger}\right]_{8} \bigotimes g^{\dagger}\right]_{0}|\Omega\rangle .
\end{aligned}
$$

The resulting TDA equation for the hybrid mass $M$ is $\left\langle\right.$ hybrid $\left.\left|\left[H, q^{\dagger} \bar{q}^{\dagger} g^{\dagger}\right]\right| \Omega\right\rangle=M\left\langle\right.$ hybrid $\left.\left|q^{\dagger} \bar{q}^{\dagger} g^{\dagger}\right| \Omega\right\rangle$.

This projects the hybrid meson wave equation, which is pictorially represented in Fig. 1, onto the three body Fock basis. Unlike our pion application, the $q \bar{q}$ pair is now in a color octet and the TDA is sufficient since the Hamiltonian, as well as exact QCD, does not conserve the chiral color octet current (we specifically calculated no difference in the more elaborate, chiral symmetry preserving hybrid RPA calculation, see below). The relevant angular momenta $(\mathrm{am})$ are the $q, \bar{q}$ spins coupled to an intermediate $S$, and the gluon spin with its orbital am $L_{+}$(with respect to the $q \bar{q} \mathrm{~cm}$ ) coupled to intermediate $l$. Coupling $l$ with $L_{-}$(the orbital $q \bar{q} a m$ ) yields $L$ which combines with $S$ giving the total am $J$. The complete wavefunction in the hybrid $\mathrm{cm}$ has form

$$
\begin{aligned}
& F_{\lambda_{g} \lambda_{q} \lambda_{\bar{q}}}^{J P C}\left(\mathbf{q}_{+}, \mathbf{q}_{-}\right) \\
& =\sum_{l L_{-} L_{+} L S m_{+} m_{-}} F_{l L_{-} L_{+} L S}^{J P C}\left(\left|\mathbf{q}_{+}\right|,\left|\mathbf{q}_{-}\right|\right) \\
& \quad \times Y_{L_{+}}^{m_{+}}\left(\hat{\mathbf{q}}_{+}\right) Y_{L_{-}}^{m_{-}}\left(\hat{\mathbf{q}}_{-}\right) \\
& \quad \times(-1)^{\lambda_{g}}\left\langle L_{+} m_{+} 1-\lambda_{g} \mid l m_{l}\right\rangle\left\langle L_{-} m_{-} l m_{l} \mid L m_{L}\right\rangle \\
& \quad \times\left\langle\frac{1}{2} \lambda_{q} \frac{1}{2}-\lambda_{\bar{q}} \mid S m_{S}\right\rangle \\
& \quad \times(-1)^{\frac{1}{2}-\lambda_{\bar{q}}}\left\langle L m_{L} S m_{S} \mid J m_{J}\right\rangle,
\end{aligned}
$$

where $\mathbf{q}_{-}$and $\mathbf{q}_{+}$are the respective relative momentum of the $q \bar{q}$ pair and gluon (with respect to the $q \bar{q} \mathrm{~cm})$. We then impose the transversality condition, $\hat{\mathbf{k}} \cdot \boldsymbol{\alpha}(\mathbf{k})=0$, from the Coulomb gauge constraint which eliminates states with $L_{+}=1$ and $l=0$. For pure $S$ waves the lightest hybrid states will then have $J^{P C}=1^{+-}, 0^{++}, 1^{++}$and $2^{++}$. These are nonexotic states which will mix with conventional mesons and hinder hybrid identification. For exotic states one $P$ wave is necessary and we calculate the lightest corresponds to $L_{+}=1$ since the $L_{-}=1$ excitation is energetically more expensive due to quark repulsion in the octet channel. This generates the exotic states $1^{-+}$, $3^{-+}$and $0^{--}$.

Instead of solving the formidable TDA nonlocal equations (effectively a 12-dimensional problem in momentum space), we evaluate the hybrid mass variationally using an exponential radial wavefunction for each of the two independent momentum variables. In the center of momentum frame the matrix elements reduce to 9-dimensional integrals that we evaluate numerically using the Monte Carlo code VEGAS. We then perform searches for minima on the energy surface in the different angular momentum channels. Our final results and key findings of this Letter are displayed in Figs. 2 and 3.

Note from Fig. 2 the clear agreement between our predictions and the lattice and flux tube results for both light and charmed hybrid states. This agreement sharply contrast with the BNL measurements which strongly suggests that the observed exotic states are not hybrids. To confirm our result is not an artifact of the variational method, we have reproduced our conventional meson and glueball exact TDA spectra to within a few percent. We also varied the least constrained model parameter $\alpha_{s}$, from 0.2 to 0.4. Related, we even performed a more extensive RPA variational calculation and took the chiral limit $\left(m_{u}=\right.$ $m_{d} \rightarrow 0$ ), finding only miniscule change in the hybrid mass, consistent with the nonconservation of the chiral color octet charge discussed above. The culmination of our model sensitivity study produced at most a $10 \%$ hybrid mass variation indicated by the box in Fig. 2 . 


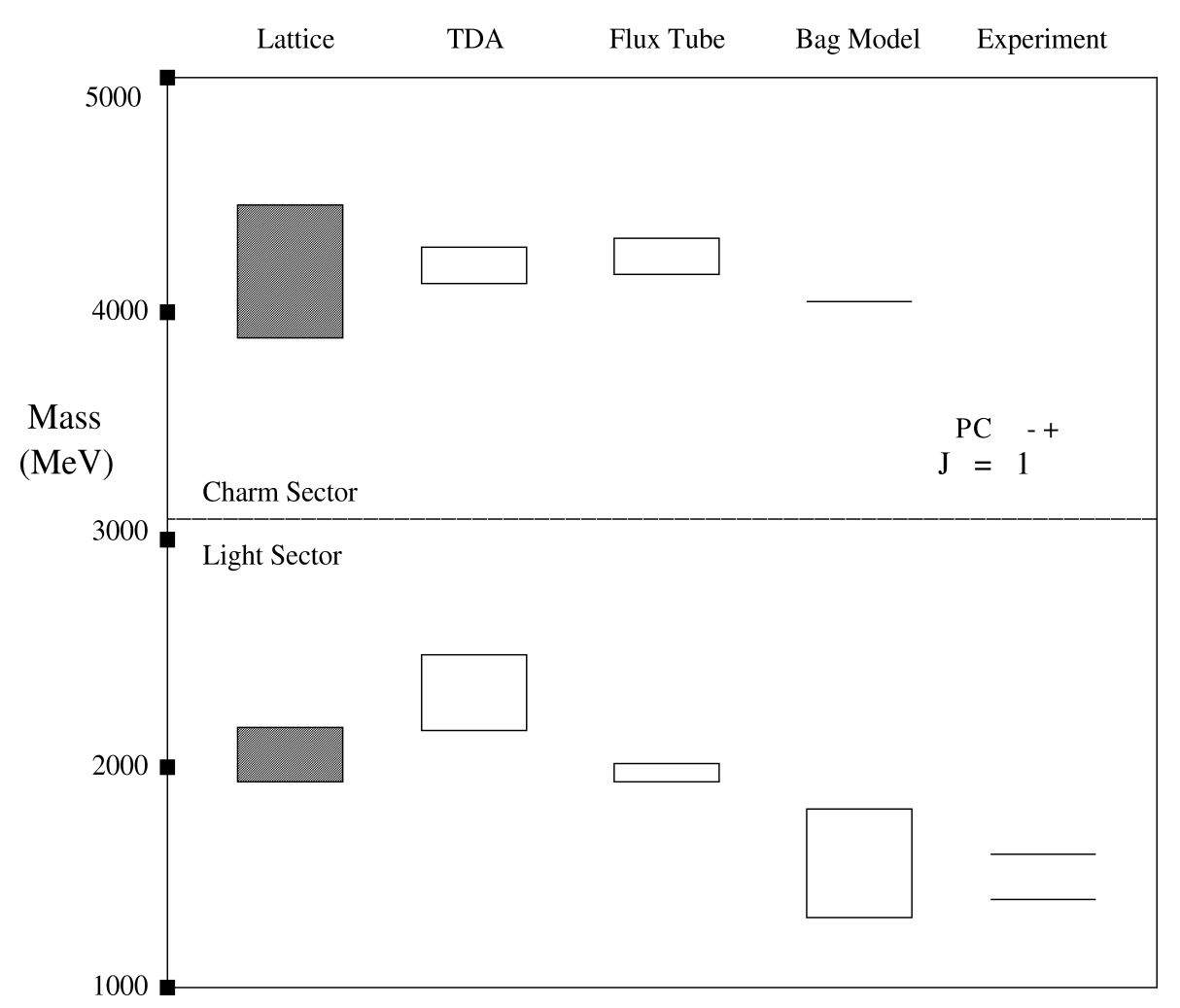

Fig. 2. Comparison of exotic $1^{-+} u / d$ and $c$ hybrid masses with alternative theories and data.

Since four quark states $q \bar{q} q \bar{q}$ can also have exotic quantum numbers, one can make simple estimates yielding exotic masses between 1 and $2 \mathrm{GeV}$ for quarks in color singlet configurations. This is consistent with a recent unitary quark model calculation [17] which also concluded that the observed $1^{-+}$states are indeed predominately meson-meson resonances.

We also calculated the lightest nonexotic hybrid (ground state) to have mass slightly above $2 \mathrm{GeV}$. It is interesting to note that this state has $J^{P C}=1^{+-}$ in contrast to certain heavy hybrid, quenched lattice ground state results [4] which find near degeneracies among several negative parity states. This was first noted by Ref. [18] which used a similar constituent model in the heavy (static) quark limit. However, the heavy quark lattice simulations do not include quark spin and until this is included the ground state hybrid quantum numbers, as well as related level ordering, remains unclear [19]. Further, another lattice calculation [3] studying orbital hybrid excitations concluded that the ground state quantum numbers were likely to be $1^{+-}$in agreement with our work. This study noted that the degeneracies in the static framework would be broken by quark spin-orbit effects which shift the exotic levels and also mix nonexotic and conventional $q \bar{q}$ states. They were also able to infer the level splitting, yielding the above conclusion, using propagating quarks on the lattice. There is less, but still some, uncertainty in level order within our model as well since our spin dependent interaction is incomplete. Although further level ordering study is necessary, the thrust of this work is the exotic hybrid and improved spin effects will not alter our conclusion that the lightest is above $2 \mathrm{GeV}$.

In Fig. 3 we compare our full model spectrum to data for the well studied, believed to be gluon rich, $1^{--}, J / \psi$ system to provide an explanation for the anomalous overpopulation of observed states [20] with respect to quark model predictions. Whereas previous constituent calculations, using only $S$ waves, could 


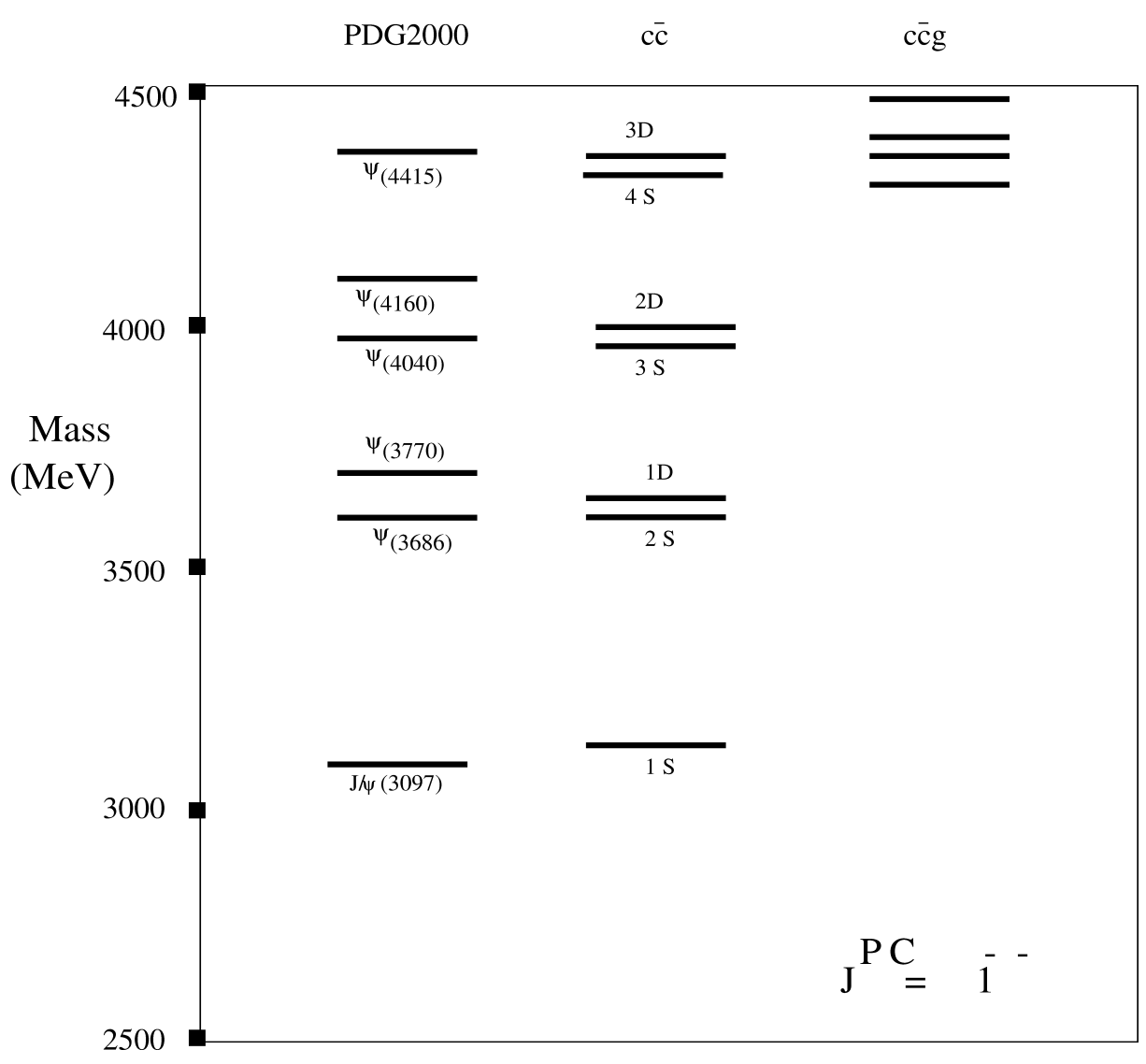

Fig. 3. TDA theory for conventional $(c \bar{c})$ and hybrid $(c \bar{c} g)$ states compared to the observed $1^{--} J / \psi$ spectrum (PDG2000) from Ref. [20].

only account for 3 of the known 6 charmonium levels, we now predict $7 c \bar{c}$ states in addition to $4 c \bar{c} g$ hybrids. Further, Ref. [20] lists an additional charmonium level $\psi(3836)$ assigned $J^{P C}=2^{--}$which also agrees well with our $D$ wave prediction (not shown). Notice that by simply including $D$ waves we have resolved the "overpopulation" problem. In general all of the $1^{-+}$states, both $c \bar{c}$ and $c \bar{c} g$, will mix and a more elaborate calculation is in progress. However, our current result is already sufficient to conclude that simple level counting (density of states) arguments will probably not be effective in identifying charmed hybrid states.

Finally, we mention a novel color octet effect leading to an isospin splitting since it only affects the $I=0$ states. This is the annihilation process depicted in Fig. 1 corresponding to $q \bar{q} \rightarrow g \rightarrow q \bar{q}$ for the $L_{-}=0$, spin aligned color octet quark pair. Octet quarkonium is the QCD analogue to ortho positronium and the annihilation interaction raises all $I=0$ light hybrid states by roughly $300 \mathrm{MeV}$ when the $q \bar{q}$ spins are aligned $(S=1)$.

Summarizing, our large-scale diagonalizations of an effective Coulomb gauge Hamiltonian provide a reasonable, comprehensive description of the meson, lattice glueball and lattice hybrid meson spectra. Further, our composite $J / \psi$ spectrum is now also in much better agreement with data, especially in terms of density of states. It is important to note that our quark/gluon unified approach essentially entails only one pre-determined dynamical parameter. Finally, and perhaps most significant, our reaffirmation of lattice and flux tube $1^{-+}$masses indicates that the recently observed exotic states are not hybrids. Based upon preliminary estimates and other independent studies it is more likely that these resonances are four quark 
states and more rigorous, higher quark Fock state calculations are in progress.

\section{Acknowledgements}

We thank NERSC for providing Cray J-90 CPU time. F.L.E. acknowledges SURA-Jefferson Lab for a graduate fellowship. This work was partially supported by grants DOE DE-FG02-97ER41048 and NSF INT-9807009.

\section{References}

[1] G.S. Adams et al., E852 Collaboration, Phys. Rev. Lett. 81 (1998) 5760;

D.R. Thompson et al., E852 Collaboration, Phys. Rev. Lett. 79 (1997) 1630.

[2] T. Barnes, F.E. Close, F. de Viron, J. Weyers, Nucl. Phys. B 224 (1983) 241.

[3] P. Lacock, C. Michael, P. Boyle, P. Rowland, Phys. Rev. D 54 (1996) 6997.

[4] C. McNeile, INFN Workshop on Hadron Spectroscopy, Frascati Physics Series 15 (1999) 13, hep-lat/9904013.
[5] T. Manke, I.T. Drummond, R.R. Horgan, H.P. Shanahan, Phys. Rev. D 57 (1998) R3829.

[6] K.J. Juge, J. Kuti, C.J. Morningstar, Nucl. Phys. Proc. Suppl. 83 (2000) 304.

[7] N. Isgur, J. Paton, Phys. Rev. D 31 (1985) 2910.

[8] T. Barnes, F.E. Close, E.S. Swanson, Phys. Rev. D 52 (1995) 5242 ;

E.S. Swanson, Nucl. Phys. Proc. Suppl. 86 (2000) 393;

L. Burakovsky, P.R. Page, hep-ph/0007199.

[9] K. Chetyrkin, S. Narison, Phys. Lett. B 485 (2000) 145.

[10] N. Brambilla, Nucl. Phys. Proc. Suppl. 86 (2000) 389.

[11] A. Le Yaouanc et al., Z. Phys. C 28 (1985) 309.

[12] F.J. Llanes-Estrada, S.R. Cotanch, Phys. Rev. Lett. 84 (2000) 1102.

[13] F.J. Llanes-Estrada, S.R. Cotanch, hep-ph/0101078.

[14] A.P. Szczepaniak, E.S. Swanson, C.-R. Ji, S.R. Cotanch, Phys. Rev. Lett. 76 (1996) 2011.

[15] N.H. Christ, T.D. Lee, Phys. Rev. D 22 (1980) 939.

[16] F.J. Llanes-Estrada, S.R. Cotanch, P.J. Bicudo, J.E.F.T. Ribeiro, A.P. Szczepaniak, hep-ph/0008212.

[17] S.M. Gerasyura, V.I. Kochin, Phys. Rev. D 62 (2000) 014008.

[18] E.S. Swanson, A.P. Szczepaniak, Phys. Rev. D 59 (1999) 014035.

[19] C. Morningstar, private communication.

[20] Eur. Phys. J. C 15 (2000) 1-878. 